\title{
Implementing Spectral Deconvolution into the Spectrum-Imaging Mode : A New Step Towards Combined High Spatial and Energy-Resolution EELS
}

\author{
A. Gloter, A. Douiri, M. Tencé, D. Imhoff, O. Stéphan and C. Colliex \\ Laboratoire de Physique des Solides (UMR CNRS 8502), Bâtiment 510, Université Paris- \\ Sud, 91405 Orsay (France)
}

In a STEM, the sub-nanometer incident probe is scanned under digital control to explore the specimen pixel per pixel, with typical step increments of a few angströms. For each position, a whole EELS spectrum encompassing an energy domain of variable width, can be acquired on a parallel detector. The whole set of spatially resolved spectra, thus generated, constitutes a complete 3D spectrum-imaging data volume [1]. Over the past few years, major progresses involving the detector efficiency and speed acquisition of EELS spectra, have been realized, using a newly developed optically coupled CCD camera [2]. When associated to the rapidly growing capabilities of modern desk computers, new perspectives can be investigated.

In order to improve the EELS energy resolution, which is presently limited by the natural width of the primary beam, by the spectrometer aberration, by the detector point spread function and by the instabilities of all electronic components, several routes can be explored. In particular, spectral deconvolution routines have shown to be quite helpful to improve energy resolution. We have recently demonstrated the potential of using a Richardson-Lucy algorithm, relying on an iterative calculation of the maximum likelihood solution [3]. The method requires the recording on the $2 \mathrm{D}$ image on the $\mathrm{CCD}$ detector of the point spread function of the whole system (source + spectrometer + detector), i.e. a non saturated image of the zero-loss peak in vacuum. The CCD image of the EELS spectrum over an energy-loss range containing the edges of interest, is recorded using similar experimental conditions. A further step has been to implement this routine into the spectrum-imaging mode. Practically, when one wants to explore the variation of EELS fine structures across a selected nanostructure, a series of 2D CCD images of spectra corresponding to the different probe positions, are recorded together with a reference zero-loss function. Up to now, the acquisition and processing tasks for improving the energy resolution of all spectra in a sequence, have only been performed on line scans. But there is no doubt that all present technical limitations will rapidly be solved and the deconvolution process routinely be performed on larger spectrum-images.

Two examples, see figs. 1 and 2, demonstrate the present state of performance with high energy resolution, in the $0.3 \mathrm{eV}$ range, on spectra recorded at typically $0.3 \mathrm{~nm}$ steps (a typical probe diameter in these experiments is $0.6 \mathrm{~nm}$ ). They concern an isolated $\mathrm{BN}$ nanotube (evolution of the $\mathrm{B} \mathrm{K}$ edge) and a thin $\mathrm{SrTiO}_{3}$ tunnel junction (evolution of the $\mathrm{Ti}_{23}$ edge) between half metallic LSMO thin films in a spin switch based on the colossal magnetotransport properties of such devices (see [4,5] for an extended discussion of the information carried in the evolution of the $\mathrm{Mn} \mathrm{L}_{23}$ and $\mathrm{O} \mathrm{K}$ edges). Obviously, these developments will become yet more fruitful when the measurements are accomplished with a sub-angström incident probe as available in the most recent $\mathrm{C}_{\mathrm{s}}$ corrected STEM instruments [6]. 
[1] C. Jeanguillaume and C. Colliex, Ultramicroscopy 28 (1989) 252

[2] M. Tencé and P. Ballongue, private communication (2002)

[3] A. Gloter et al., to be published, Ultramicroscopy (2003)

[4] F. Pailloux et al., Phys. Rev. B 66 (2002) 14417

[5] L. Samet et al., submitted (2003)

[6] P. E. Batson et al., Nature 418 (2002) 617

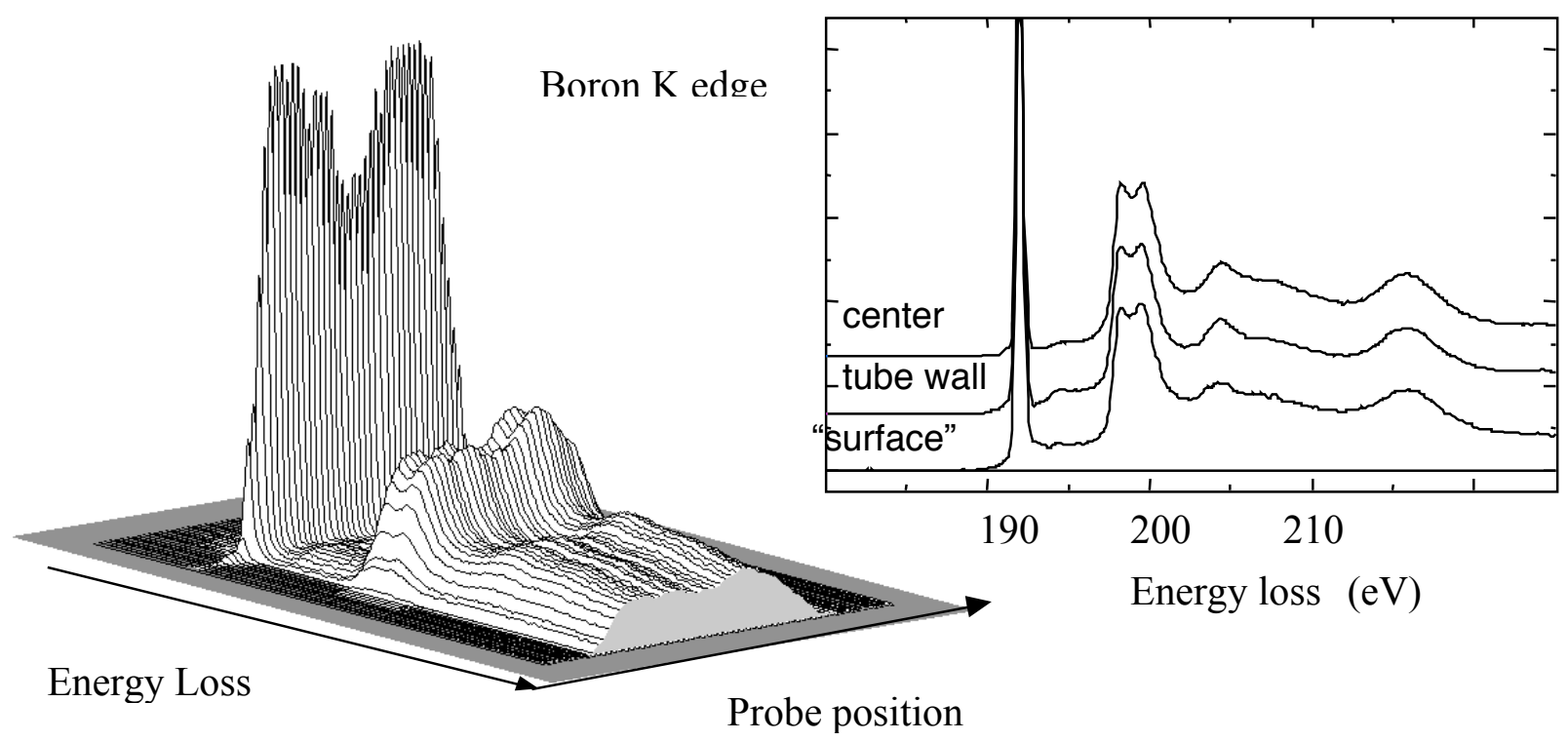

Figure 1 : Series of deconvoluted spectra (boron K edge after background subtraction) across an individual $\mathrm{BN}$ nanotube (10 $\mathrm{nm}$ outer diameter and $4 \mathrm{~nm}$ inner diameter) exhibiting a * line of $0.36 \mathrm{eV}$ FWHM and changes in relative intensity and fine structures from center to outer wall.

Figure 2 : Series of deconvoluted spectra (Ti L lines) with $0.4 \mathrm{eV}$ FWHM on the $\mathrm{L}_{3} \mathrm{t}_{2 \mathrm{~g}}$ line $\left(\mathrm{a}_{1}\right)$, across a $\mathrm{SrTiO}_{3}$ tunnel junction of $5.5 \mathrm{~nm}$ thickness grown between two thin $\mathrm{La}_{2 / 3} \mathrm{Sr}_{1 / 3} \mathrm{MnO}_{3}$ films for a spin injection device (specimen courtesy of CNRS-Thales laboratory)

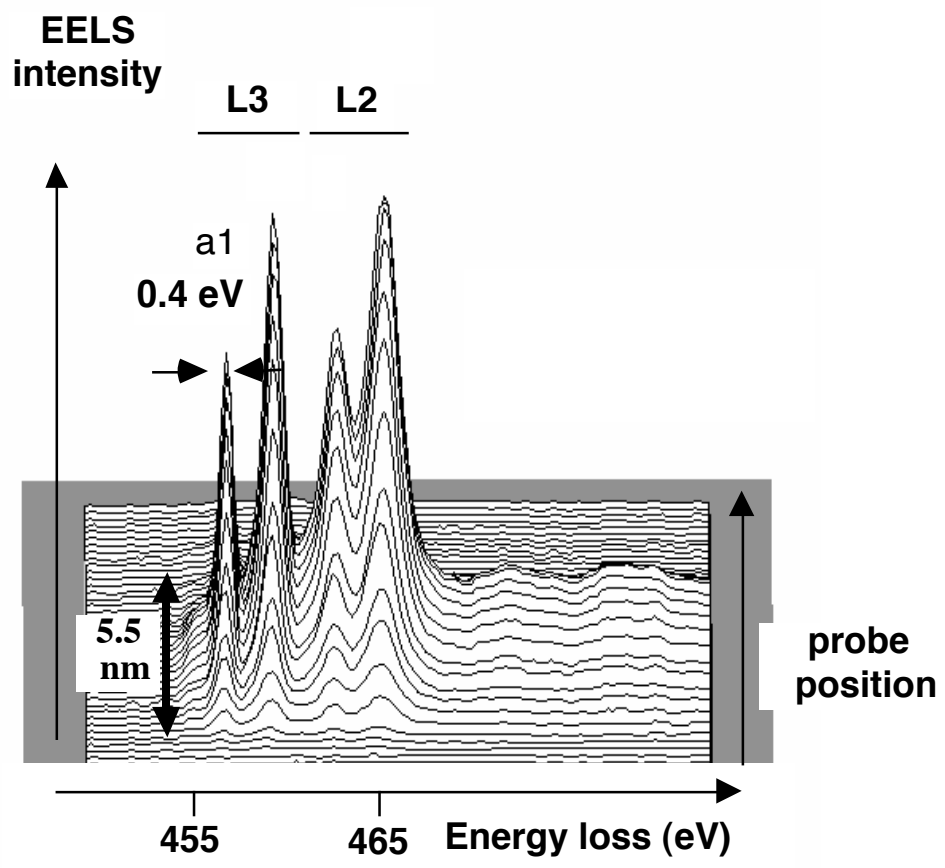

